\title{
A New Method for the Design of Slot Antenna Arrays: Theory and Experiment
}

\author{
Sebastien Clauzier ${ }^{1}$, Said M. Mikki ${ }^{2}$, Atif Shamim ${ }^{3}$, and Yahia M. M. Antar ${ }^{1}$, \\ ${ }^{1}$ Royal Military College of Canada, Electrical and Computer Engineering Department, Station Forces, Kingston, ON, Canada. \\ ${ }^{2}$ University of New Haven, Electrical and Computer Engineering \& Computer Science Department, West Haven, CT, USA, \\ ${ }^{3}$ King Abdullah University of Science and Technology, Thuwal 23955-690, Kingdom of Saudi Arabia.
}

\begin{abstract}
The present paper proposes and validates a new general design methodology that can be used to automatically find proper positions and orientations of waveguide-based radiating slots capable of realizing any given radiation beam profile. The new technique combines basic radiation theory and waveguide propagation theory in a novel analytical model that allows the prediction of the radiation characteristics of generic slots without the need to perform full-wave numerical solution. The analytical model is then used to implement a low-cost objective function within a global optimization scheme (here genetic algorithm.) The algorithm is then deployed to find optimum positions and orientations of clusters of radiating slots cut into the waveguide surface such that any desired beam pattern can be obtained. The method is verified using both fullwave numerical solution and experiment.
\end{abstract}

Index Terms-Infinitesimal dipole model, slotted waveguide, Optimization program.

\section{INTRODUCTION}

Slot arrays are among the earliest technologies originally developed for applications involving wireless communications [1], [2]. Here, the idea is to avoid direct feeding circuits at each array element by utilizing instead only one common "substructure" supporting an illumination field impinging on the slots' open areas. According to the classic surface equivalence theorem, the impinging electric field will generate an equivalent magnetic current that can efficiently radiate into free space [1]. In this case, no special matching circuit and phase shifters are needed at each radiating slot antenna. The challenge, however, is how to control the relative amplitude and phase excitation of each slot in order to achieve a desired radiation beam pattern. The present paper proposes and validate a general methodology that can help the antenna and communication engineer in finding the best locations and orientations of each slot in order to satisfy such general requirements imposed on the radiation pattern.

For the sake of definiteness, we concentrate in this paper on waveguide-based slot arrays, though the method proposed here is quite general and can be applied to any radiating slot configuration. Slotted waveguides have found many applications in radar and communication systems due to their low-profile design requirements, mechanical robustness, good efficiency, and the relative ease of realization compared with other conventional antenna arrays [3]-[5]. Early in the beginning of the slotted waveguide technology, methods have been proposed to determine the positions/orientations of the slots in order to achieve a given radiation pattern [3], [6]. More recently, the original approach by Elliot [6] was used to propose optimization-based algorithms for slot antenna arrays [7].

These methods, however, are too complicated and appear to be cumbersome to realize. The basic idea in Elliot [3], [6] was to consider each half-wavelength waveguide section with one slot as a basic "slot module" with variable load impedance attached to it. Afterwards, an equivalent circuit model for this "slot module" is found, which includes mutual coupling with other slots. The designer will then try to use a combination of proper load impedances and excitations at each "slot module" in order to realize the desired excitation on the slot itself. Using conventional array theory, the radiation pattern of the slot array is then implemented by the array factor of classical dipole arrays.

The present paper proposes a completely different approach to the problem of slot array design that is much easier to implement and also eventually leading to more direct practical realization. In comparison with other methods, only one port is involved with one matching circuit. The waveguide is terminated with an absorbing load and therefore the complexity of having phase shifters and variable loads attached at several locations in the systems is completely avoided. The design method proposed here works very well as long as the slot length is off resonance. It is found that by small modification of the slot length below the resonance length, there is no need to introduced additional measurements to account for mutual coupling effects.

\section{THEORITICAL MODEL FOR SLOT ARRAYS AND VALIDATION}

It is well-known that a slot is equivalent to a magnetic dipole, with the following relation [1], [2]

$$
\mathbf{M}=-2 \vec{n} \times \mathbf{E}
$$

where $\mathbf{M}$ is the magnetic current, $\mathbf{E}$ the electric current and $\vec{n}$ the normal vector. Once the slot has been replaced by a magnetic current, the current itself can be discretized by replacing it by an array of magnetic infinitesimal dipole (MIDs). The infinitesimal dipole model method has been used successfully in many applications, ranging from mutual coupling and antenna modeling [8] to near-field focusing [9] and MIMO antenna design [10]. In the present paper we propose applying the method to develop a slot array design method. 
An array of $N$ magnetic infinitesimal dipoles possesses the following field expression [8]-[11]

$$
\begin{gathered}
\boldsymbol{H}(\boldsymbol{r})=\sum_{n=1}^{N} \frac{1}{4 \pi \varepsilon_{0}}\left(k_{0}^{2} \cdot\left(\boldsymbol{n}_{\boldsymbol{n}} \times \boldsymbol{p}_{\boldsymbol{n}}\right) \times \boldsymbol{n}_{\boldsymbol{n}}\right) \cdot \frac{e^{-j k_{0} r_{n}}}{r_{n}} \\
\boldsymbol{E}(\boldsymbol{r})=\sum_{n=1}^{N} \frac{c_{0} k_{0}^{2}}{4 \pi}\left(\boldsymbol{n}_{\boldsymbol{n}} \times \boldsymbol{p}_{\boldsymbol{n}}\right) \cdot \frac{e^{-j k_{0} r_{n}}}{r_{n}}
\end{gathered}
$$

where,

$$
\boldsymbol{n}_{\boldsymbol{n}}=\left(\frac{x-x_{d}(n)}{r_{n}}, \frac{y-y_{d}(n)}{r_{n}}, \frac{z-z_{d}(n)}{r_{n}}\right)^{T}
$$

is the unit vector from the location of the $n$th ID to an observation point, and

$$
\boldsymbol{p}_{\boldsymbol{n}}=A_{d}(n) \cdot e^{j \varphi_{d(n)}} \cdot\left(\begin{array}{c}
\cos \left(\phi_{d}(n)\right) * \sin \left(\theta_{d}(n)\right) \\
\sin \left(\theta_{d}(n)\right) * \sin \left(\phi_{d}(n)\right) \\
\cos \left(\theta_{d}(n)\right)
\end{array}\right)
$$

is the moment of the $n$th dipole, $A_{d}(n)$ is the $n$th dipole (complex) excitation, and

$$
r_{n}=\sqrt{\left(x-x_{d}(n)\right)^{2}+\left(y-y_{d}(n)\right)^{2}+\left(z-z_{d}(n)\right)^{2}}
$$

is the distance from the center of the infinitesimal dipole to the observation point, $k_{0}$ is the wave-number in free space $\left(k_{0}=\frac{2 \pi}{\lambda}\right), \varepsilon_{0}$ is the vacuum permittivity $\left(\varepsilon_{0} \cong 8.85 \times 10^{8} \mathrm{~F} /\right.$ $m), c_{0}$ is the celerity of the light $\left(c_{0} \cong 3 \times 10^{8} \mathrm{~m} / \mathrm{s}\right) . \phi_{d}$ and $\theta_{d}$ are the orientation angles of the ID and $x_{d}, y_{d}, z_{d}$ their positions. In the present paper, each slot will be modeled by an array of 5 MIDs. The slots will have a length slightly different from $\lambda / 2$. Moreover, for simplicity, we will not consider orientations in the specific examples to be presented below (i.e., $\phi_{d}=\theta_{d}=0$ ). Using this approach, radiation from each slot can be given in a complete analytical form.

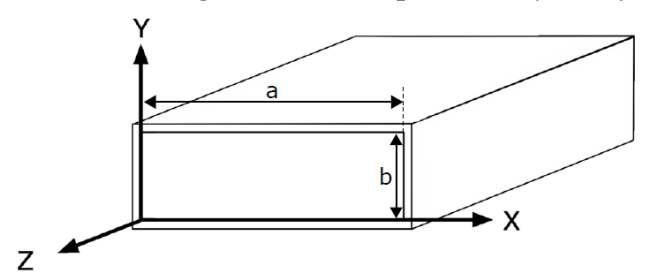

Fig. 1. Schematic view of a rectangular waveguide.

It remains to find the excitation of each group of MIDs used to model each slot. Since the variation of the field in a waveguide is well-known, it is expected that for narrow slots, the near field produced within the slot is close to the propagating mode excited for conventional un-slotted waveguide. If we consider only the dominant $\mathrm{TE}_{10}$ mode, the phase variation along the waveguide represented in Fig 1, will be given by [11]

$$
\varphi_{w g}=\frac{2 \pi f}{c} \sqrt{1-\left(\frac{f_{c}}{f}\right)^{2}} \cdot z
$$

while the amplitude of the field along the $\mathrm{x}$-axis will follow the variations [11]

$$
A(x) \propto H_{z}=\frac{j \pi Y_{T E}}{\beta_{g} a} \cdot \cos \left(\frac{\pi x}{a} \cdot e^{j\left(\omega t-\beta_{g} z\right.}\right)
$$

Here, $Y_{T E}$ is the dominant mode wave admittance, $f$ is the operational frequency ( $f=10 \mathrm{GHz}$ in the present work), $f_{c}$ is the cut-off frequency $\left(f_{c}=c / 2 a\right.$ for the $\mathrm{TE}_{10}$ mode $), a$ is the broadside length of the waveguide, and $\beta_{g}=2 \pi / \lambda_{g}$ is the guided wave wavenumber. In this paper, we consider slots inserted in the broadside of the waveguide. We remind that in order to obtain an 'in-phase' field, we have to alternate the slot position on both side of the axis of its broadside as shown in Fig. 2.

The theoretical model above was verified in the following way. A full wave model of the waveguide with slots was constructed using full-wave solvers (CST and WIPL-D). A near field probe was placed inside the slot to measure the field amplitude and phase. The field variation across slots of various lengths were record and compared with the theoretical formulas (4) and (5), upon which they were found to agree. The MID model equations (2) and (3) were then used to predict the far field produced by the slots based on the excitation levels predicted by (4) and (5). The pattern obtained this way was compared with the far field produced by the fullwave solver. Excellent agreement again was observed (see Section IV for numerical examples.) The model is capable of taking mutual coupling into account (because of the good agreement with full-wave data). However, it was noticed that when the slot length is exactly at resonance length, the model becomes inaccurate. To solve this problem, all slots will be assigned a length slightly off resonance length.

With equations (4) and (5) we can predict theoretically the amplitude and the phase inside each slot as function of its position/orientation. Together with the MID's expression (2), These equations will be used in the optimization process to determine analytically the field produced for each modification of the position and orientation of each slot obtained by the search algorithm.

\section{PROPOSED METHOD}

The method can be summarized as follows

1. First, we don't search for any circuit model for the slots involved. Instead, we work directly with the field generated in the slot within the array environment. This field will be measured by a nearfield probe in the simulation in order to verify the theoretical model.

2. Based on the surface equivalence theorem, the electric field in the slot will be converted into a radiating magnetic current.

3. Using the analytical fields' expressions of the magnetic infinitesimal dipole (MID), we approximate 
the equivalent radiating slot magnetic current by a small number of MIDs placed in the position and the orientation of the actual slot.

4. Using waveguide theory, the mode field is used directly to provide information on how the excitations of the various MIDs in the model of each slot will be chosen. This provides a complete analytical model of the entire waveguide-slot array that is as accurate as full-wave solutions, making its subsequent use in extensive optimization algorithms possible.

5. The far field produced by the slot array is computed analytically from the formula of the MID and a square error function is formed to compare with a desired radiation pattern.

6. Finally, through a global optimization algorithm (GA in this case), we search for the best location and orientation of the slots resulting in the best fit of the radiation pattern.

Based on the theoretical model of the previous section, the radiation pattern produced by the slot array can be written as a single function $\mathbf{E}^{\mathrm{rad}}=F\left(\mathbf{x}_{s}, \mathbf{o}_{s}\right)$ depending only on the slot's positions $\mathbf{x}_{s}$ and orientations $\mathbf{o}_{s}$. This function can now be used in an optimization algorithm to minimize the square error

$$
e\left(\mathbf{x}_{s}, \mathbf{o}_{s}\right)=\int_{\left|\mathbf{E}^{\text {rad }}\right|>\left|\mathbf{E}^{\text {desired }}\right|}\left|\mathbf{E}^{\mathrm{rad}}\left(\theta, \phi ; \mathbf{x}_{s}, \mathbf{o}_{s}\right)-\mathbf{E}^{\text {desired }}(\theta, \phi)\right|^{2} d \Omega,
$$

Where integration is performed only for the angles where $\left|\mathbf{E}^{\text {rad }}(\theta, \phi)\right|>\left|\mathbf{E}^{\text {desired }}(\theta, \phi)\right|$ (In other words, the error is set to zero when $\left|\mathbf{E}^{\mathrm{rad}}(\theta, \phi)\right|<\left|\mathbf{E}^{\text {desired }}(\theta, \phi)\right|$, i.e., when the obtained beam is already below the target mask.

Here, $\mathbf{E}^{\mathrm{rad}}\left(\theta, \phi ; \mathbf{x}_{s}, \mathbf{o}_{s}\right)$ is the far field produced by the array with slot's positions $\mathbf{x}_{s}$ and orientations $\mathbf{o}_{s}$, while $\mathbf{E}^{\text {desired }}(\theta, \phi)$ is the desired (target) radiation pattern. The objective is to find the optima $\mathbf{x}_{s}^{*}$ and $\mathbf{o}_{s}^{*}$ such that

$$
\left.e^{*}\right|_{\mathbf{x}_{s}, \mathbf{o}_{s}=\mathbf{x}_{s}^{*}, \mathbf{0}_{s}^{*}}=\min _{\mathbf{x}_{s}, \mathbf{o}_{s}} e\left(\mathbf{x}_{s}, \mathbf{o}_{s}\right)
$$

In the remaining parts of this paper, the proposed method above will be validated and illustrated through the design, implementation, and testing of a concrete array example.

\section{COMPUTATIONAL AND EXPERIMENTAL VALIDATION OF THE PROPOSED DESIGN METHOD}

In order to verify the analytical model of the slotted waveguide, we considered first a simple (not optimized) example: slotted waveguide consisting of an array of 10 slots with an inter-element distance of $\lambda_{g} / 2$ and at a same distance from the medial axis (Fig. 2). This slotted waveguide was modeled by an array of 50 MIDs (5 MIDs for each slot). The amplitude and the phase for each infinitesimal dipole was determined with the help of (4) and (5). The far-field radiation patterns based on the analytical model and the one computed by full-wave solver (CST Microwave Studio) are compared in Fig. 3 for two cut-planes $(\varphi=\pi / 2$ et $\varphi=\pi / 4)$ with very good agreement. The directivity for the analytic model is equal to $15.6 \mathrm{dBi}$ and for the simulated waveguide is equal to $16.7 \mathrm{dBi}$.

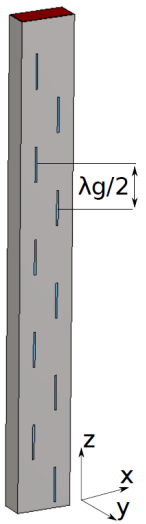

Fig. 2. View of a standard slotted waveguide.

Next, we try to optimize the positions of the slots on the waveguide in order to reach a specific radiation pattern. The specifications for the far-field radiation pattern chosen for the sake of illustration in this paper are given in table I.

TABLE I. MAIN CHARACTERISTICS OF THE RADAITION PATTERN.

\begin{tabular}{|c|c|}
\hline Characteristics & Value \\
\hline HPBW (degrees) & 10 \\
\hline Side Lobe Level $(\mathrm{dB})$ & -10 \\
\hline Beam direction (degrees) & 15 \\
\hline
\end{tabular}

As mentioned previously, we have used the genetic algorithm (GA) in order to optimize the position of each slot, modeled by an array of infinitesimal dipole. After 100 iterations, the optimized $(x, z)$ found positions are given in table II. Fig 4 shows the optimized positions of the slot on a designed slotted waveguide.

TABLE II. OPTIMIZED POSITION OF THE SLOTS.

\begin{tabular}{|c|c|c|}
\hline Slot & $\mathbf{x}(\mathbf{m m})$ & $\mathbf{z}(\mathbf{m m})$ \\
\hline 1 & 2.96 & 0 \\
\hline 2 & -1.48 & 19.9 \\
\hline 3 & 3.71 & 30.58 \\
\hline 4 & -2.41 & 40 \\
\hline 5 & 3.15 & 60 \\
\hline 6 & -1.85 & 75.3 \\
\hline 7 & 2.96 & 91.7 \\
\hline 8 & -1.67 & 100 \\
\hline 9 & 2.59 & 120 \\
\hline 10 & -3.15 & 130.64 \\
\hline
\end{tabular}



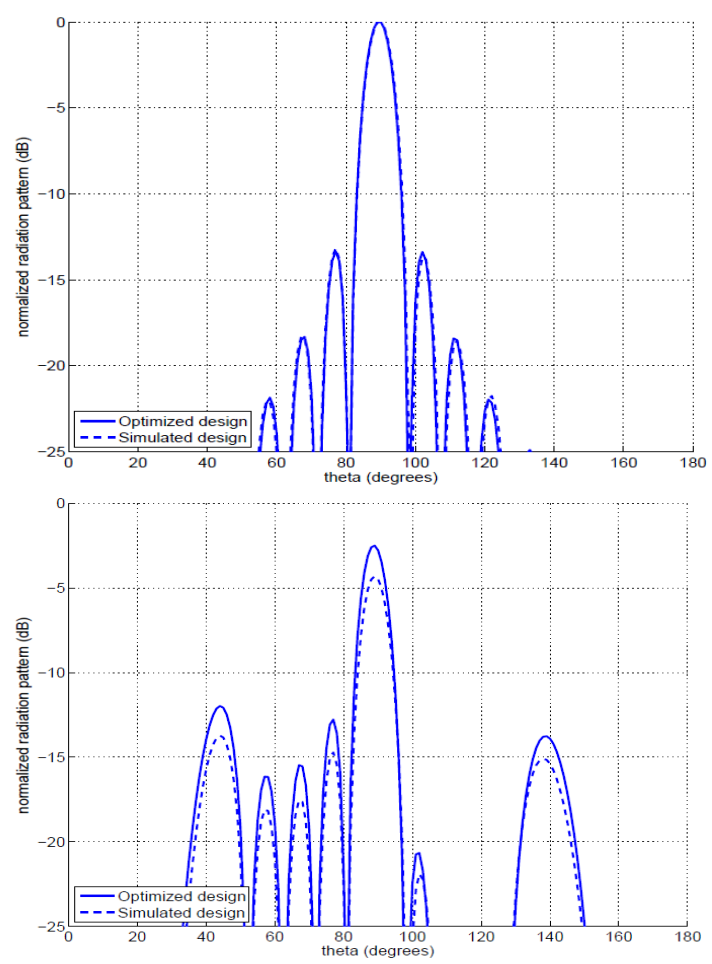

Fig. 3. Far-field radiation pattern for the analytic array of infinitesimal dipole and for tha actual waveguide simulated on CST MWS for $\varphi=\pi / 2$ (top) and $\varphi=\pi / 4$ (bottom)

In Fig.5, the radiation patterns along $\theta$ for $\varphi=\pi / 2$ due to both the analytical model MID model and the actual antenna simulated on CST MWS are compared. Again a very good agreement is observed. All the desired specifications defined in the table I have been reached. The slight observable differences are due to edge radiation in the simulation, which was not considered in the theoretical MID-based model. The directivity computed by the analytical model is equal to $12.9 \mathrm{dBi}$ and for the simulated waveguide is equal to $14 \mathrm{dBi}$. By increasing the length of the waveguide, edge radiation effects can be ignored and in this case the two predictions of the directivity can be made closer to each other.
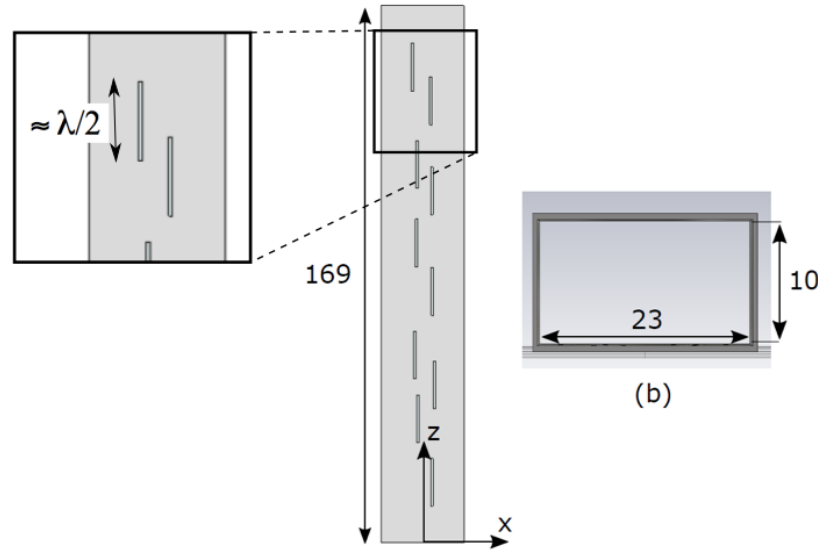

(b)

(a)

Fig. 4. View of the optimized slotted waveguide (side(a) and top(b)).

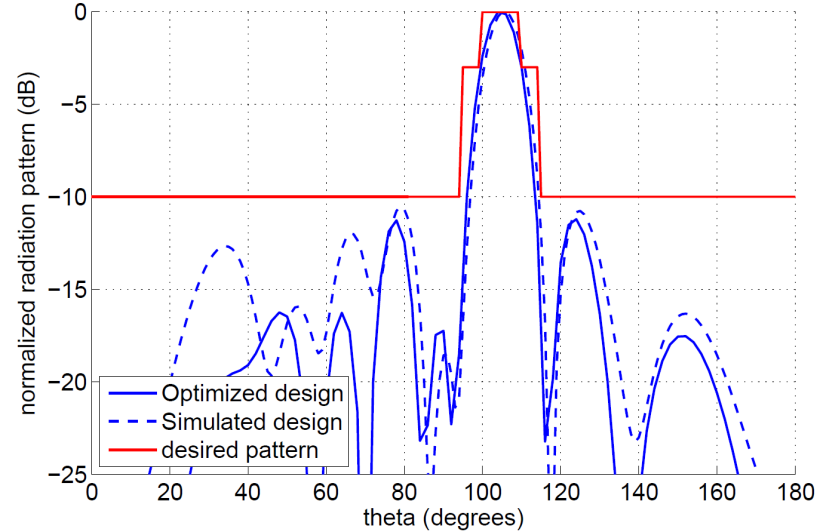

Fig. 5. Radiation pattern of the optimized array of infinitesimal dipole (solid line) and of the simulated slotted waveguide on CST MWS (dashed line).

Based on the design data obtained after the optimization process, the slotted waveguide was physically realized in the lab. A 3D model printed using Object Connex 3D printer by Stratasys was internally metalized to achieve a measured performance of the antenna as close to simulation as possible. The critical dimensions of the waveguide antenna i.e. "a", "b", and the positions and locations of the slots were extracted from CST model and the flanges were added on the input and output ports of the slotted waveguide in order to excite the waveguide and absorb the non-radiated RF signal, respectively. Final prototype is shown in Fig. 6.
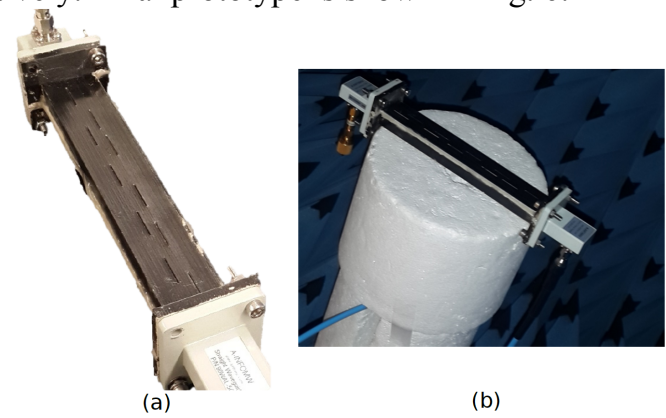

(b)

Fig. 6. Fabricated waveguide antenna (a) and measurement setup (b).

The slotted waveguide antenna was excited with the help of an SMA-to-waveguide adapter while the other end of the waveguide was terminated into matched $50 \Omega$ load so that any non-radiated RF signal could be absorbed by that port. In this way, the matched load acts the same way as a perfect absorber, which is needed here in order to avoid reflections from port 2. The measurement setup is shown in Fig. 6(b). The slotted waveguide antenna performance was measured in anechoic chamber within the frequency range $8 \mathrm{GHz}-10 \mathrm{GHz}$. In Fig. 7, we provide the radiation pattern along $\theta$ for $\varphi=$ $\pi / 2$ for the analytic model, the simulated model and the measured waveguide.

As can be seen in Fig. 7, there is a good agreement between simulation and measurement. Table III highlights the key points of the comparison between theory and measurement. Deviations can be explained by the presence of high $\varepsilon_{r}=2.8$ in the $3 \mathrm{D}$ printed material of $1.5 \mathrm{~mm}$ thickness outside the 
metallic coating of the slotted waveguide antenna (which in the case of theory is the air medium with $\varepsilon_{r}=1$ ).

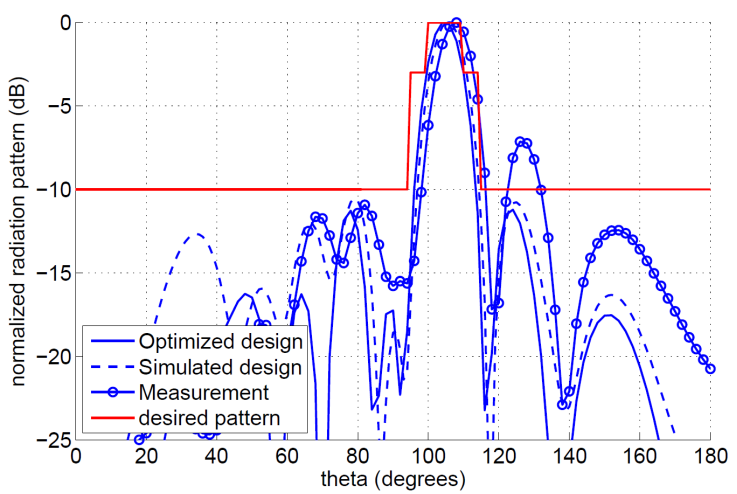

Fig. 7. Radiation pattern of the optimized array of infinitesimal dipole (solid line) and of the slotted waveguide simulated on CST (dashed line)).

TABLE III. COMPARISON OF THE OPTIMIZED, THE SIMULATED AND THE MEASURED RESPONSE OF THE SLOTTED WAVEGUIDE.

\begin{tabular}{|l|c|c|c|c|}
\hline Property & $\begin{array}{c}\text { Desired } \\
\text { pattern }\end{array}$ & $\begin{array}{c}\text { Analytic } \\
\text { model }\end{array}$ & Simulation & Measurement \\
\hline $\begin{array}{l}\text { Beam } \\
\text { direction }\end{array}$ & $15^{\circ}$ & $15^{\circ}$ & $16^{\circ}$ & $18^{\circ}$ \\
\hline HPBW & $10^{\circ}$ & $10.5^{\circ}$ & $10^{\circ}$ & $10.5^{\circ}$ \\
\hline SLL & $-10 d B$ & $-11 d B$ & $-10.5 d B$ & $-7.5 d B$ \\
\hline SLL & \multicolumn{5}{|c|}{ Good agreement } \\
\hline
\end{tabular}

Concerning the gain, the simulated gain at $10 \mathrm{GHz}$ is equal to $12 \mathrm{~dB}$ and the measured gain is equal to $4 \mathrm{~dB}$. The important difference between the simulated and measured gain is due again to the presence of the high $\varepsilon_{r}=2.83 \mathrm{D}$ printed material of $1.5 \mathrm{~mm}$ thickness outside the metallic coating of the slotted waveguide antenna. Indeed this material induces a shift in the operational frequency with a maximum gain around $8.8 \mathrm{GHz}$. A retro-simulation on CST has shown that if we consider this material, the gain in simulation is equal to $4.6 \mathrm{~dB}$ which is close to the measured one. With a gain of $12 \mathrm{~dB}$, the efficiency of the waveguide is about $50 \%$. As said previously, this efficiency is the result of a tradeoff on the length of the slot between the efficiency and the accuracy of the theoretical model.

\section{CONCLUSION}

We proposed a generalized new methodology for the design of slotted-waveguide antenna arrays based on the use of infinitesimal magnetic dipoles to model radiation from slots using analytical formulas as accurate as full-wave simulation. The dominant waveguide propagating modal fields where used directly in the analytical model to predict the variation of the excitations across each slot models using proper groups of infinitesimal magnetic dipoles. The fact that the entire radiation pattern of the waveguide slot array can now be modeled by a simple analytical model allowed us to use expensive optimization algorithms to search for the best positions of the slots capable of achieving a desired radiation pattern. The method was found to work very well for small and large slots provided they are off the resonance length. The proposed theoretical analytical model was validated by direct comparison with full-wave analysis. Afterwards, the model was used to form a square error function in order to search for the best fit of the obtained pattern to desired radiation characteristics. The optimization algorithm provides positions and/or orientation of slots, which were used to build a physical model of the system. Measurement of the radiation pattern confirmed the design method and provided a validation of the proposed methodology. The present approach is much simpler than existing methods since here no special treatment of mutual- and self-impedances at each port is needed. Moreover, only one port, the input waveguide port, is involved for the whole system, and there is no need to vary excitation voltages by external circuits like attenuators and phase shifters. Moreover, mutual coupling effects are shown to be taken automatically into consideration using our method without special treatment except at slots of resonant length.

\section{REFERENCES}

[1] C. A. Balanis, Antenna Theory: Analysis and Design. WileyInterscience, 2005.

[2] J. Volakis, Antenna Engineering Handbook, Fourth Edition. McGrawHill Companies,Incorporated, 2007.

[3] R.S. Elliott. "An Improved Design Procedure for Small Arrays of Shunt Slots”, IEEE Trans. Antennas and Propagation, Vol. 31, pp. 48-53, January 1983.

[4] Casula, G.A.; Mazzarella, G.; Montisci, G., "Shaped beam synthesis technique for linear arrays of waveguide longitudinal slots," in Antennas and Propagation Society International Symposium (APSURSI), 2012 IEEE, vol., no., pp.1-2, 8-14 July 2012.

[5] Clauzier, S.; Avrillon, S.; Le Coq, L.; Himdi, M.; Colombel, F.; Rochefort, E., "Slotted waveguide antenna with a near-field focused beam in one plane," in Microwaves, Antennas \& Propagation, IET, vol.9, no.7, pp.634-639, 5 15, 2015.

[6] R. S. Elliott and L. A. Kurtz, "The design of small slot arrays," IEEE Trans. Antemas Propagat., vol. 26, pp. 216219, Mar. 1978.

[7] Oraizi, H. and Noghani, M.T., "Design and Optimization of WaveguideFed Centered Inclined Slot Arrays," in Antennas and Propagation, IEEE Transactions on , vol.57, no.12, pp.3993-3997, Dec. 2009

[8] Mikki, S.M.; Kishk, A.A., "Theory and Applications of Infinitesimal Dipole Models for Computational Electromagnetics," in Antennas and Propagation, IEEE Transactions on, vol.55, no.5, pp.1325-1337, May 2007

[9] Sebastien Clauzier, Said M. Mikki, and Yahia M. M. Antar, "Design of near-field synthesis arrays through global optimization," IEEE Trans. Antennas Propagat., vol. 63, no. 1, pp. 151-165, January 2015.

[10] S. Clauzier, S. Mikki, and Y. Antar, "A generalized methodology for obtaining antenna array surface current distributions with optimum cross-correlation performance for MIMO and spatial diversity applications," IEEE Antennas and Wireless Propagation Letters, vol. 14, pp. 1451-1454, 2015.

[11] Carlo G. Someda, Electromagnetic Waves, CRC Press, 2006. 